\title{
Opponent-process theory: The interaction of trials, intertrial interval, and the presence of evoking stimuli
}

\author{
DONALD R. YELEN \\ Washburn University, Topeka, Kansas
}

\begin{abstract}
Two predictions from Solomon's (1980) opponent-process theory were tested in the present experiment. The first prediction was that there would be an interaction between trials, or the number of times that an opponent process is aroused, and the presence or absence of the stimuli used to evoke the primary process. This prediction was supported $(\mathrm{p}<.01)$ by GSR data collected while college students were either squeezing a hand dynamometer or resting. The second prediction, that the length of the intertrial interval would affect the interaction between trials and the presence of the stimuli that evoke the primary process, was not supported.
\end{abstract}

Solomon (1980) and his colleagues (Hoffman \& Solomon, 1974; Solomon \& Corbit, 1974) have proposed an opponent-process theory to account for a variety of motivational phenomena. This theory is based on the notion of a single negative feedback loop. The loop is initiated when a primary affective process is aroused by an adequate stimulus of some sort, such as an operant reinforcer, a UCS, or an innate releaser. The primary process then arouses a competing process that functions to oppose and suppress the affective or hedonic state generated by the onset of the primary process.

The opposing processes are postulated to have different properties. The primary process, arbitrarily designated $a$, is essentially a stable unconditioned reaction whose strength is fixed by the intensity, duration, and quality of the evoking stimulus. The opponent process, or $b$, on the other hand, is strengthened through use and weakened through disuse. Specifically, it is postulated that increasing its use will result in process $b$ 's having (1) a shorter latency of response to process $a$, (2) a faster rate of approach to the asymptote, (3) a higher asymptote, and (4) a slower rate of decay when process $a$ has been terminated.

The theory also postulates that affective or hedonic State A will be manifested if the absolute strength of process $a$ is greater than the absolute strength of process $b$, and that affective State B will be manifested if the opposite is true. Finally, the theory postulates that the intensity of the affective state that is manifested will be determined by the absolute difference between the opposing processes.

The present experiment was designed, first, to determine whether the intensity of an affective state was a function of the joint effects of trials, that is, the number of times process $b$ is aroused and the presence or absence

\footnotetext{
The author's mailing address is: Department of Psychology, Wash-
} burn University, Topeka, KS 66621. of the stimuli used to evoke process $a$. An interaction between these two variables is expected to occur, on the basis of the theory's postulate stating that the strength of process $a$ is stable or fixed by the evoking stimuli, whereas the strength of process $b$ increases with use. When the stimulus that evokes process $a$ is present, an increase in the strength of process $b$ with trials would result in a decrease in the intensity of the affective state because the absolute difference between the opposing processes would be reduced. When, however, the evoking stimulus is absent, an increase in the strength of process $b$ with trials would result in an increase in the intensity of an affective state because the absolute difference between the opposing processes would be increased.

The present experiment was also designed to determine whether the interaction described above was affected by the length of the interval between trials. It was assumed that disuse was a direct function of the length of the intertrial interval, and therefore, increasing the time between trials was expected to retard the growth of process $b$ because disuse is postulated to weaken process $b$. If the growth of process $b$ is retarded by the length of the intertrial interval, then the interaction between the number of trials given and the presence or absence of the stimuli used to evoke process $a$ should be suppressed or eliminated when relatively long intertrial intervals are used, but should not be affected when relatively short intervals are used.

\section{METHOD}

A primary affective process was produced in the present study by requiring college students to squeeze a hand dynamometer as hard as they could for $30 \mathrm{sec}$. It was assumed that this procedure would result in an aversive affective process similar to those produced by the activities used by Solomon (1980) and his colleagues to explain opponent-process theory, that is, activities such as parachuting, sauna bathing, and jogging.

The intensity of the affective state produced by squeezing the hand dynamometer was assumed to be directly related to ordinary psychophysi- 
cal measures such as the galvanomic skin response (GSR). This assumption is consistent with Solomon's (1980) use of heart rate data to illustrate the dynamics of opponent processes.

\section{Procedure}

College students were instructed that the experiment was concerned with the effects of muscle tension on physiological and psychological processes. A hand dynamometer was then adjusted for each student, and its use was demonstrated. The students were instructed to use the right hand to squeeze the device as hard as possible when they were asked to do so during the course of the experiment. The students were to continue squeezing as hard as they could until the command to rest was given. At this time, the students could sit back and relax. Following these instructions, electrodes for recording GSR were attached to the first and third fingers of each student's left hand. It was explained that the electrodes were used to measure physiological changes associated with muscle tension.

After the instructions had been given; electrodes attached, and questions answered, the students were assigned randomly to either a 30 - or a 60-sec intertrial interval condition. All students were then administered a series of five trials. Each trial was initiated by the experimenter's giving the command "Squeeze." Each trial ended $30 \mathrm{sec}$ later when the experimenter gave the command "Rest." Students in the 30-sec intertrial condition were given $30 \mathrm{sec}$ between the "Rest" command on one trial and the "Squeeze" command on the following trial. This interval was $60 \mathrm{sec}$ in the 60 -sec condition. The first trial for the 30 -sec condition was started 2 min after the first trial for the 60 -sec condition in order to ensure that both groups of students would spend the same amount of time in the apparatus.

The dependent measures, GSRs averaged over $5 \mathrm{sec}$, were recorded on two occasions during Trials 1 and 5 . The first recording on each of these trials was made $20 \mathrm{sec}$ after the command "Squeeze" had been given. This measure was assumed to reflect the intensity of the affective state when the evoking stimulus was present. The second GSR recording on each trial was made $20 \mathrm{sec}$ after the students had been given the "Rest" command. This measure was assumed to reflect the intensity of the affective state when the evoking stimulus was absent.

\begin{abstract}
Apparatus
The GSRs, in micromhos of skin conductance, were recorded using conductive gel and silver/silver-chloride finger electrodes attached with Velcro strips to the first and third fingers of each student's left hand. These recordings were sent to a data file on an Apple II+ disk via a J\&J Model IG-3/T-68 GSR preamp and recorder, a DIG-880 serial interface, and Humanistic Chart Recorder System software. The students were required to squeeze a LaFayette hand dynamometer.
\end{abstract}

\section{Subjects}

A total of 46 introductory psychology students participated in this experiment. Half of the students, 11 males and 12 females, were assigned randomly to the 30 -sec intertrial interval condition; the remaining students, 10 males and 13 females, were assigned to the 60 -sec condition.

\section{RESULTS}

Figure 1 presents the mean GSR for the students in the combined 30- and 60-sec conditions when the students were either squeezing the hand dynamometer (evoking stimulus present) or resting (evoking stimulus absent) during Trials 1 and 5. Inspection of this Figure 1 shows that the mean GSR decreased from Trial 1 to Trial 5 when the students were squeezing the hand dynamometer, but that the reverse was true when they were resting, that is, that the mean GSR increased from Trial 1 to Trial 5 in the condition. The results of an analysis of variance supported these observations. More specifically, the interaction of the number of trials administered, one or five,

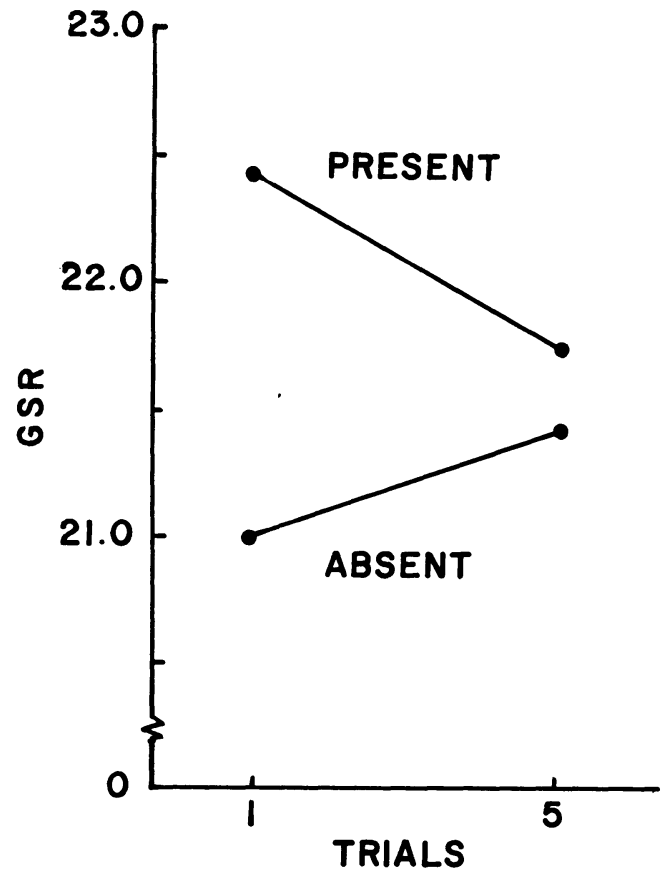

Figure 1. Mean GSR for the subjects in the combined 30- and 60sec conditions when the evoking stimulus was either present (squeezing the hand dynamometer) or absent (resting) during Trials 1 and 5.

with the presence or absence of the evoking stimulus was significant $[\mathrm{F}(1,44)=8.64, \mathrm{p}=.005]$. These results, then, support the first prediction tested in the present experiment.

The second prediction tested in the present study, however, was not supported by the data. That is, varying the length of the intertrial interval between 30 and $60 \mathrm{sec}$ did not affect the interaction between the number of trials administered and the presence or absence of the evoking stimuli. In fact, the analysis of variance indicated that the $F$ value for the triple interaction (intertrial interval $\times$ number of trials administered $\times$ presence or absence of stimuli) was less than 1.0.

\section{DISCUSSION}

The present experiment tested two predictions based on the postulate from Solomon's (1980) opponent-process theory stating that process $b$ is strengthened through use and weakened by disuse. Use was defined by the number of trials administered and disuse by the length of the intertrial interval. The results supported the idea that use would strengthen process $b$ and are thus consistent with findings reported by Solomon (1980), his colleagues (Starr, 1978), and independent investigators (Piliavin, Callero, \& Evans, 1984).

The results of the present experiment, however, did not support the idea that disuse would weaken process $b$. The variable that needs to be examined in this context is the length of the intertrial intervals used to define disuse. The 30- and 60 -sec intervals used in the present study were chosen simply to have two points that were a reasonable distance apart on a temporal continuum. It is quite conceivable that both of these intervals fall within what Solomon (1980) called the "critical decay duration of process $b$." Solomon (1980) stated that process $b$ can be strengthened by use "if and only if it is aroused at intervals less than the critical decay duration" (p. 710). It follows from this argument that 
process $b$ can be weakened only if it is aroused at intervals greater than the critical decay duration. Further experimentation is needed to determine whether the length of the intertrial intervals used in the present study failed to have a weakening effect on process $b$ simply because they were too short and fell within the critical decay duration, or whether the original postulate needs to be revised or modified in some way.

\section{REFERENCES}

Hoffman, H. S., \& Solomon, R. L. (1974). An opponent-process theory of motivation: III. Some affective dynamics in imprinting. Learning and Motivation, 5, 149-164.
Pillavin, J. A., Callero, P. L., \& Evans, D. E. (1984). Addiction to altruism? Opponent-process theory and habitual blood donation. Journal of Personality and Social Psychology, 43, 1200-1213.

Solomon, R. L. (1980). The opponent-process theory of acquired motivation: The costs of pleasure and the benefits of pain. American Psychologist, 36, 691-712.

Solomon, R. L., \& CoRBIT, J. D. (1974). An opponent-process theory of motivation: I. Temporal dynamics of affect. Psychological Review, 81, 119-145.

STARR, M. D. (1978). An opponent-process theory of motivation: VI. Time and intensity variables in the development of separation-induced distress calling in ducklings. Journal of Experimental Psychology: Animal Behavior Processes, 4, 338-355.

(Manuscript received for publication October 8, 1984.) 